\title{
Changes in Pre- and Post-Electroconvulsive Therapy Serum Myostatin Levels in Patients with Treatment Resistant Depression
}

\author{
Cemil Çelik, Abdullah Bolu, Taner Öznur, Mehmet Sinan Aydın, Yusuf Tokgöz, Özcan Uzun \\ Department of Psychiatry, Gülhane Faculty of Medicine, University of Health Sciences, Ankara, Turkey
}

\begin{abstract}
Objective: Myostatin is a growth factor which is investigated regarding musculoskeletal system. Albeit its effect on muscle mass is known, it is considered likely having other unknown effects as well, particularly on central nervous system. With this study, it is aimed to find out that what type of effect electroconvulsive therapy (ECT) does on myostatin in patients with treatment resistant depression.

Methods: Twenty-nine patients with treatment resistant major depression and thirty healthy volunteers were included in the study. Pre- and post-ECT levels of myostatine were compared; also this results were compared to healthy controls. Results: For 29 patients with treatment-resistant major depression, the pre-treatment mean myostatin level was $0.95 \pm 0.32$ $\mathrm{ng} / \mathrm{ml}$ and post-therapy myostatin level was $11.05 \pm 6.97 \mathrm{ng} / \mathrm{ml}$. As a result of this study, it is found that ECT affects serum myostatin levels to a significant degree $(\mathrm{t}=4.17, p<0.05)$. It is also found that there was a significant relation between serum myostatin levels and depression scores $(r=0.392, p<0.05)$.

Conclusion: With the present study and similar ones, it can be understood that how ECT achieves its effectiveness biologically.
\end{abstract}

KEY WORDS: Electroconvulsive therapy; Depression; Myostatine.

\section{INTRODUCTION}

Myostatin, also known as growth and differentiation factor-8 (GDF-8), a member of the transforming growth factor- $\beta$ superfamily and regulated probably by microRNAs, plays an important role in the control of musculo-skeletal development as a negative regulator of muscle growth via Smads and non-Smad signal pathways. ${ }^{1-4)}$ In addition to its muscle growth inhibition, myostatin has recently been demonstrated to function in protein, fat and glucose metabolisms. ${ }^{5)}$ Blocking myostatin expression results in increased insulin sensitivity associated with increased adiponectin secretion from adipose tissue. ${ }^{6)}$

There are some findings showing that myostatin has a potential role in neurotransmission, particularly in dop-

Received: July 11, 2017 / Revised: August 10, 2017

Accepted: September 5, 2017

Address for correspondence: Cemil Çelik, MD

Department of Psychiatry, Gülhane Faculty of Medicine,

University of Health Sciences, Etlik-06018, Ankara, Turkey

Tel: +90-312-304-4501, Fax: +90-312-304-4507

E-mail: cemil.celik@sbu.edu.tr

ORCID: https://orcid.org/0000-0002-1021-8762 amine regulation, ${ }^{7)}$ but findings about this relationship are indirect. However, there is no data regarding myostatin in evaluation of efficacy of treatments such as antidepressants or electroconvulsive therapy (ECT) in psychiatric diseases, particularly in depression, so far. On the other hand, there are studies regarding depression and antidepressant effectiveness of adiponectin in the adipose tissue, induced by myostatin. In a study of Guo et al. ${ }^{8)}$ it was demonstrated that there was a relation between adiponectin and stress, anxiety and depression tendency. At the same time, there are data showing that adiponectin has antidepressant effectiveness. ${ }^{9)}$

Myostatin is a molecule, mostly thought to be associated with chronic heart failure and musculo-skeletal system. It was shown that myostatin level increased in patients with chronic heart failure and it has changed in parallel with the exercise. ${ }^{10,11)}$ It was found that its level was changed depending on the type of exercise in normal healthy individuals. ${ }^{12,13)}$ As the molecule is directly thought to be associated with musculo-skeletal system, it has been subject to studies related to muscular activities. ${ }^{14)}$ However, in an application such as ECT, in which muscular activ-

(ㄷ) This is an Open-Access article distributed under the terms of the Creative Commons Attribution Non-Commercial License (http://creativecommons.org/licenses/by-nc/4.0) which permits unrestricted non-commercial use, distribution, and reproduction in any medium, provided the original work is properly cited. 
ities continue and muscle contractions present despite use of muscle relaxants, it is not known how plasma level is affected.

It is known that ECT effects levels of brain-derived neurotrophic factor (BDNF), nerve growth factor, neuropeptide $Y$, somatostatine. ${ }^{15-19)}$ Despite of these findings, it is still unknown that "which molecule has main role in ECT's effect mechanism”. ${ }^{20)}$

In this study, we aimed to investigate the effectiveness of ECT on serum myostatin levels in patients with treatment resistant major depression. Moreover, we also aimed to investigate the relation between plasma myostatin levels and response to ECT.

\section{METHODS}

\section{Participants}

In this study, the patients with treatment resistant major depression, who received ECT in inpatient settings in Department of Psychiatry, Gülhane Military School of Medicine between 2015 and 2016, are evaluated. Thirty patients with treatment resistant major depression (12 males and 18 females) and a control group of 30 healthy people (12 meals and 18 females) are included in the study. Patients were evaluated according to the Structured Clinical Interview for DSM-IV Axis I Disorders (SCID-I) and twelve patients with comorbid psychiatric diseases were excluded from the study.

One female patient is excluded from the study as side effect secondary to ECT developed. Treatment resistance is defined as no response to at least three antidepressant therapies with appropriate dose and duration according to stage III definiton of Thase and Rush. ${ }^{21)}$ The drugs likely to affect seizure threshold (antidepressants or antipsychotics) were not given to patients during ECT. Benzodiazepine for sedation purpose was given in cases of requirement.

Patients and controls groups underwent a medical history taking and a physical assessment. They were screened for acute infectious diseases by measuring body temperature, erythrocyte sedimentation rate, C-reactive protein and urinary culture. Exclusion criteria were any additional axis I or axis II DSM-IV diagnosis, current pregnancy, acute or chronic infections within the last month, autoimmune, allergic, neoplastic, or endocrine diseases (thyroid and other endocrine dysfunctions) or other acute physical disorders, including surgery or cardiac or cere- bral infarction within the last 3 months. Patients, who took any drug including nonsteroidal anti-inflammatory drugs or oral contraceptives in the last 6 weeks, were also excluded. Healthy volunteers were also interviewed and in addition to the above exclusion criteria, those with no lifetime or current diagnosis of any psychiatric disorders were included in the control group.

Socio-demographic (age, gender, level of education, and marital status) and clinical conditions of the subjects were examined by using a data query form. Major depressive episode was diagnosed using the DSM-5. The severity of depression was evaluated with the 21-item Beck Depression Inventory (BDI). The validity and reliability studies of the Turkish version of the form were conducted by Hisli. ${ }^{22)}$

The study was approved by Gülhane Military Medical Academy, Ethical Statement of Clinical Researches (50687469-1491-42-15/SEK.1677). Written informed consent form was obtained from each participant.

\section{Application and Sample Collection}

The patients with treatment resistant major depression, for whom ECT was planned, were screened according to SCID-I for additional diagnoses. Necessary psychometric assessments, biochemical examinations and consultations were done by the treatment team. The BDI scale was applied twice; before and after the ECT application to patients who met the criteria to be included in the study without any contraindication to ECT, and venous blood samples were drawn to measure the myostatin levels. In the control group, venous blood samples were drawn only once to measure the myostatin levels.

\section{ECT Application}

ECT was carried out by a team of an anesthesiologist, a psychiatrist, a psychiatric nurse and anesthesia technicians at the ECT unit of the Department of Psychiatry. Subsequent to a fasting period (8-12 hours) and after the standard anesthesia monitoring, patient-specific doses of propofol $(0.75-1 \mathrm{mg} / \mathrm{kg})$ for anesthesia induction and the muscle relaxant succinylcholine $(1 \mathrm{mg} / \mathrm{kg})$ were administered intravenously to all patients by an anesthesiologist.

Bilateral ECT was administered. The number of ECT applications in our study was between 8 and 15 for a frequency of thrice a week, and this number could be in- 
creased or decreased by the patient's clinical response. Electrical stimulus was provided by a Mecta Spectrum 5000Q ECT (Portland, OR, USA) device. In the ECT application, the formation of 25 seconds of long-term convulsions was targeted by administering square wave-type pulses of 550 to $800 \mathrm{~mA}$ (frequency, $30-70 \mathrm{~Hz}$ ). The seizure threshold was terminated at the first treatment. Stimuli were given with increasing intensity until a generalized seizure was induced. All patients were oxygenated by positive pressure ventilation via mask until resumption of spontaneous respiration.

\section{Serum Myostatin Levels}

Peripheral venous blood samples were collected in serum tubes from all participants at 6:00 to 9:00 am. All samples were allowed to clot before centrifuged at $1,000 \times g$ for 15 minutes and were stored at $-80^{\circ} \mathrm{C}$ until analysis. Serum concentrations of myostatin (GDF-8) were determined in duplicate with commercially available enzyme-linked immunosorbent assay (ELISA kits; Wuhan Eiaab Science Co. Ltd., Wuhan, China). The minimum detectable level of myostatin was $0.156 \mathrm{ng} / \mathrm{ml}$. Intra- and interassay coefficients of variation were $6.9 \%$ and $9.7 \%$, respectively.

\section{Statistical Analyses}

Kolmogorov-Smirnov test was used to check if the sample distribution was normal. Inter-group comparison of the demographic variables was done using an ANOVA for continuous variables and the chi-square test for categorical variables. Correlations between variables were assessed with the Pearson's correlation coefficient. Paired $t$ test was used to investigate changes in myostatin levels before and after treatment. Data are presented as mean standard deviation. All tests were two-tailed with a sig- nificance level set at 0.05 . The rate was set to $\alpha=0.05$ and $p$ values equal to or lower than this value were considered statistically significant. SPSS version 15.00 (SPSS Inc., Chicago, IL, USA) was used for data analysis.

\section{RESULTS}

\section{Socio-demographic Characteristics}

The study was concluded by 29 patients and 30 healthy volunteers. There was no difference between the two groups in terms of age, marital status, education and gender. The groups were also similar in terms of socio-demographic features (Table 1).

\section{Clinical Scales and Changes with Treatment}

Pre-ECT BDI scores were found to be $42.13 \pm 8.14$; while post-ECT BDI scores were $15.89 \pm 6.89$. The difference between the two measurements was found to be statistically significant $(\mathrm{t}=17.28, p<0.05)$.

\section{Myostatin Measurements}

For 29 patients with treatment-resistant major depression, the pre-treatment mean myostatin level was $0.95 \pm 0.32 \mathrm{ng} / \mathrm{ml}$, while in the control group the mean myostatin level was $0.87 \pm 0.21 \mathrm{ng} / \mathrm{ml}$. There was no statistically significant difference between pre-therapy myostatin levels of the patient group and the control group $(\mathrm{t}=1.28, p=0.229)$. However, post-therapy myostatin level of the patient group was measured to be $11.05 \pm 6.97$ $\mathrm{ng} / \mathrm{ml}$. There was a statistically significant difference between post-therapy myostatin levels of the patient group and post-therapy myostatin levels of the control group $(\mathrm{t}=5.08 ; p<0.05)$. Accordingly, there was a statistically significant difference between in pre-therapy myostatin levels and post-therapy myostatin levels of the patient

Table 1. Socio-demographic and clinical characteristics of patient and control groups

\begin{tabular}{lccc}
\hline \multicolumn{1}{c}{ Characteristic } & Major depression $(\mathrm{n}=29)$ & Control $(\mathrm{n}=30)$ & $\mathrm{t} / \chi^{2}$ \\
\hline Sex & $12(41.4)$ & $12(40.0)$ & 0.564 \\
Male & $17(58.6)$ & $18(60.0)$ & 0.453 \\
Female & $36.66 \pm 9.70$ & $33.60 \pm 11.23$ & 0.908 \\
Age (yr) & $9.7 \pm 4.0$ & $11.2 \pm 4.3$ & 1.334 \\
Education level (yr) & $11.6 \pm 2.0(8-15)$ & 0.188 \\
No. of electroconvulsive therapy & & \\
\hline
\end{tabular}

Values are presented as number (\%) or mean \pm standard deviation (range).

t, by Student's t test; $\chi^{2}$, Pearson's chi-square test. 


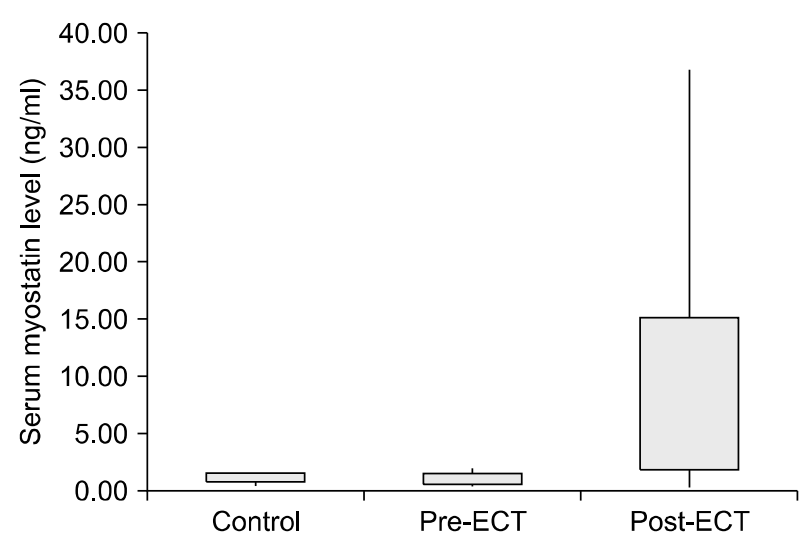

Fig. 1. Healty control group, serum myostatin levels of pre- and post-ECT patients.

ECT, post-electroconvulsive therapy.

group (t=4.17; $p<0.05)$ (Fig. 1).

\section{Relationship between BDI Scores and Myostatin and ECT}

No statistically significant relation was found between myostatin levels and BDI scores. However, statistically significant and positive correlation was found between myostatin level and change in BDI scores $(r=0.392, p<$ 0.05). There was no significant difference between numbers of ECT and myostatin levels.

\section{DISCUSSION}

In our study, pre-ECT myostatin levels were same between patients and control group, but post-ECT levels were different between these groups. Patients post-ECT miyostatin levels were significantly higher than pre-ECT. This was the first important finding of our study. The second important result is finding a significant relation between myostatin levels and depression scores.

Our study is the first one to evaluate the relation between ECT and myostatin levels. We believe that shedding light on this subject may contribute to understanding the effect mechanism of ECT. The most widely used method to observe ictal motor activity during ECT is to prevent muscle relaxant to pass through a limb of the patient (generally by placing a cuff around the right or left arm). ${ }^{23}$ This condition may be a reason for the elevation of myostatin level.

Results of the studies, performed on the subject of musculo-skeletal system and myostatin level, are contra- dictory. It was demonstrated that the elevated myostatin levels in patients with chronic heart diseases decreased after exercise training. ${ }^{11)} \mathrm{A}$ previous animal study reported that stretching and electrical stimulation to muscle, which mimic exercise training, decreased myostatin levels. ${ }^{14}$ In our study, elevation of myostatin level in the opposite way may be a result of the muscular activities during ECT being very different from the regular exercise. Because exercise training has shown inconsistent effects on myostatin expression in healthy volunteers, depending on the muscle type and the form of exercise. ${ }^{12,13)}$

It seems very strange to us that albeit there is a relation between myostatin levels and depression scores, there is no relation between numbers of ECT and myostatin levels. Yet, there is no study on effect of myostatin on mood. Myostatin is thought to increase the adiponectin synthesis in adipose tissue. ${ }^{24)}$ However, adiponectin is a protein on which studies are carried out regarding depression. AdipoR1 and AdipoR2 are adiponectin receptors in central nervous system, and they play a role in mood regulation, being effective in various parts of the brain including hippocampus. ${ }^{25,26)}$ In animal model studies, adiponectin demonstrated antidepressant-like effect. ${ }^{9,26)}$ Yet, there is no sufficient data regarding the nature of the relation between depression and myostatin. However, as said above, there is a close interaction between adiponectin and myostatin. Myostatin may indirectly affect the depressive mood through adiponectin. As result of animal model studies and growth factor studies such as BDNF and vascular endothelial growth factor, ECT is thought to increase hippocampal neurogenesis. ${ }^{27-29)}$ Hippocampal neurogenesis with anti-depressant effects has been related to adiponectin secretion. ${ }^{30)} \mathrm{ECT}$ is thought to be related to hippocampal growth and antidepressant effects and adiponectin is also thought to be a potential biological determinant. ${ }^{31)}$ We are of the opinion that myostatin has an effective role in all those effects, and it can contribute to shedding light on these steps.

Direct relation between the change in myostatin levels and the change BDI scales, which is a determinant of ECT response, and no relation between pre-ECT myostatin and $\mathrm{BDI}$ scores in this study, give rise to thought that myostatin change can be used as a determinant of response to ECT, such as adiponectin.

This study contains some limitations. First, it could be performed on a larger group of patients. Second, myo- 
statin levels could be compared between patients with treatment resistant depression and other patients with depression. Within sametime other biomarkers could be determined which effects levels of myostatin. Patients' myostatin levels could be measured several times during therapy, and it could be found out that each ECT session yielded what kind of results. We believe that particularly the studies in which adiponectin levels will be measured together with myostatin levels will be the most valuable in the future.

\section{REFERENCES}

1. Lee SJ, McPherron AC. Myostatin and the control of skeletal muscle mass. Curr Opin Genet Dev 1999;9:604-607.

2. Sharma M, Langley B, Bass J, Kambadur R. Myostatin in muscle growth and repair. Exerc Sport Sci Rev 2001;29:155-158.

3. Tsuchida K. Targeting myostatin for therapies against muscle-wasting disorders. Curr Opin Drug Discov Devel 2008; 11:487-494.

4. Kollias HD, McDermott JC. Transforming growth factor-beta and myostatin signaling in skeletal muscle. J Appl Physiol (1985) 2008;104:579-587.

5. Huang Z, Chen X, Chen D. Myostatin: a novel insight into its role in metabolism, signal pathways, and expression regulation. Cell Signal 2011;23:1441-1446.

6. Han HQ, Mitch WE. Targeting the myostatin signaling pathway to treat muscle wasting diseases. Curr Opin Support Palliat Care 2011;5:334-341.

7. Filonzi L, Franchini N, Vaghi M, Chiesa S, Marzano FN. The potential role of myostatin and neurotransmission genes in elite sport performances. J Biosci 2015;40:531-537.

8. Guo M, Li C, Lei Y, Xu S, Zhao D, Lu XY. Role of the adipose PPARy-adiponectin axis in susceptibility to stress and depression/anxiety-related behaviors. Mol Psychiatry 2017;22: 1056-1068

9. Chan JS, Li A, Ng SM, Ho RT, Xu A, Yao TJ, et al. Adiponectin potentially contributes to the antidepressive effects of Baduanjin Qigong exercise in women with chronic fatigue syndrome-like illness. Cell Transplant 2017;26:493-501.

10. Gruson D, Ahn SA, Ketelslegers JM, Rousseau MF. Increased plasma myostatin in heart failure. Eur J Heart Fail 2011;13: 734-736.

11. Lenk K, Erbs S, Höllriegel R, Beck E, Linke A, Gielen S, et al. Exercise training leads to a reduction of elevated myostatin levels in patients with chronic heart failure. Eur J Prev Cardiol 2012;19:404-411.

12. Coffey VG, Shield A, Canny BJ, Carey KA, Cameron-Smith D, Hawley JA. Interaction of contractile activity and training history on $m R N A$ abundance in skeletal muscle from trained athletes. Am J Physiol Endocrinol Metab 2006;290:E849E855.
13. Costa A, Dalloul $\mathrm{H}$, Hegyesi $\mathrm{H}$, Apor $\mathrm{P}$, Csende Z, Racz L, et al. Impact of repeated bouts of eccentric exercise on myogenic gene expression. Eur J Appl Physiol 2007; 101:427-436.

14. Russo TL, Peviani SM, Durigan JL, Gigo-Benato D, Delfino GB, Salvini TF. Stretching and electrical stimulation reduce the accumulation of $M y O D$, myostatin and atrogin- 1 in denervated rat skeletal muscle. J Muscle Res Cell Motil 2010;31: 45-57.

15. Polyakova $M$, Schroeter ML, Elzinga BM, Holiga $S$, Schoenknecht $\mathrm{P}$, de Kloet ER, et al. Brain-derived neurotrophic factor and antidepressive effect of electroconvulsive therapy: systematic review and meta-analyses of the preclinical and clinical literature. PLoS One 2015;10:e0141564.

16. Allen AP, Naughton M, Dowling J, Walsh A, Ismail F, Shorten $\mathrm{G}$, et al. Serum BDNF as a peripheral biomarker of treatment-resistant depression and the rapid antidepressant response: A comparison of ketamine and ECT. J Affect Disord 2015; 186:306-311.

17. Angelucci F, Aloe L, Jiménez-Vasquez $P$, Mathé AA. Electroconvulsive stimuli alter nerve growth factor but not brain-derived neurotrophic factor concentrations in brains of a rat model of depression. Neuropeptides 2003;37:51-56.

18. Nikisch G, Mathé AA. CSF monoamine metabolites and neuropeptides in depressed patients before and after electroconvulsive therapy. Eur Psychiatry 2008;23:356-359.

19. Kragh J, Tønder N, Finsen BR, Zimmer J, Bolwig TG. Repeated electroconvulsive shocks cause transient changes in rat hippocampal somatostatin and neuropeptide $Y$ immunoreactivity and mRNA in situ hybridization signals. Exp Brain Res 1994;98:305-313.

20. Singh A, Kar SK. How electroconvulsive therapy works?: Understanding the neurobiological mechanisms. Clin Psychopharmacol Neurosci 2017;15:210-221.

21. Thase ME, Rush AJ. When at first you don't succeed: sequential strategies for antidepressant nonresponders. J Clin Psychiatry 1997;58 Suppl 13:23-29.

22. Hisli N. Validity and reliability of Beck Depression Scale in university students. Turk J Psychol 1989;7:13-19.

23. American Psychiatric Association. The practice of electroconvulsive therapy: recommendations for treatment, training, and privileging (A task force report of the American Psychiatric Association). 2nded. Arlington:American Psychiatric Pub;2000.

24. Suzuki ST, Zhao B, Yang J. Enhanced muscle by myostatin propeptide increases adipose tissue adiponectin, PPAR-alpha, and PPAR-gamma expressions. Biochem Biophys Res Commun 2008;369:767-773.

25. Fry M, Smith PM, Hoyda TD, Duncan M, Ahima RS, Sharkey $\mathrm{KA}$, et al. Area postrema neurons are modulated by the adipocyte hormone adiponectin. J Neurosci 2006;26:9695-9702.

26. Liu J, Guo M, Zhang D, Cheng SY, Liu M, Ding J, et al. Adiponectin is critical in determining susceptibility to depressive behaviors and has antidepressant-like activity. Proc Natl Acad Sci U S A 2012;109:12248-12253. 
27. Segi-Nishida E, Warner-Schmidt JL, Duman RS. Electroconvulsive seizure and VEGF increase the proliferation of neural stem-like cells in rat hippocampus. Proc Natl Acad Sci U S A 2008; 105:11352-11357.

28. Evans SJ, Choudary PV, Neal CR, Li JZ, Vawter MP, Tomita H, et al. Dysregulation of the fibroblast growth factor system in major depression. Proc Natl Acad Sci U S A 2004;101: 15506-15511.

29. Madsen TM, Treschow A, Bengzon J, Bolwig TG, Lindvall O,
Tingström A. Increased neurogenesis in a model of electroconvulsive therapy. Biol Psychiatry 2000;47:1043-1049.

30. Yau SY, Li A, Hoo RL, Ching YP, Christie BR, Lee TM, et al. Physical exercise-induced hippocampal neurogenesis and antidepressant effects are mediated by the adipocyte hormone adiponectin. Proc Natl Acad Sci U S A 2014;111:15810-15815.

31. Sitzmann AF. Treatment-resistant cepression, obesity, and adiponectin. Michigan:University of Michigan;2015. [Thesis]. 